\title{
COMPARATIVE PHARMACOGNOSTIC EVALUATION OF MARKET SAMPLES OF PRSNIPARNI WITH GENUINE SOURCE OF URARIA PICTA (JACQ) DESV EX DC
}

\author{
Surabhi $\mathbf{M}^{1}$, Anuradha K N ${ }^{2}$ \\ ${ }^{I} P G$ Scholar \\ ${ }^{2}$ Assistant Professor, Department of Dravyaguna, Shri Dharmasthala Manjunatheshwara College of \\ Ayurveda and hospital, Hassan-573201
}

Article DOI: https://doi.org/10.36713/epra8828 DOI No: 10.36713/epra8828

\begin{abstract}
The medicinal plants and its active principles are playing a dominant role in the maintenance of human health since ancient times. The degree of threat to natural population of medicinal plants has increased, because more than $90 \%$ of medicinal plant raw material for herbal industries in India draw from natural habitat In ayurvedic medicine Dasamula plants are top traded group and their annual demand is more than1000 Metric tons, Prsniparni moola is one among the Dasamula, The degree of threat to natural population of medicinal plants has increased, because more than 90\% of medicinal plant raw material for herbal industries in India draws from natural habitat, this demand is not often met with supply of original raw drugs alone and is topped up with other species of plants that are substitutes or adulterants. This directly affects the quality and efficacy of the herbal products and the same has reflected in case of Uraria picta. Over usage, destructive harvesting and lack of cultivation have reduced the availability of Uraria picta. Inorder to characterize and compare the raw drugs used as prsniparni in market, the candidate species traded under the name Prsniparni were subjected to macroscopic and organoleptic evaluation, microscopic study, Physical and Phytochemical evaluation.
\end{abstract}

\section{INTRODUCTION}

The medicinal plants and its active principles are playing a dominant role in the maintenance of human health since ancient timesThe annual demand of botanical raw drugs in India was estimated to be 3,19,500 Metric tons during the year 2005-2006 amounting trade value of Rs. 1,069 crores. ${ }^{1}$ The degree of threat to natural population of medicinal plants has increased, because more than $90 \%$ of medicinal plant raw material for herbal industries in India draw from natural habitat. This demand is not often met with supply of original raw drugs alone and is topped up with other species of plants that are substitutes or adulterants. As a result, the quality of herbal products becomes compromised. Natural sources of medicinal plants are often unable to meet demand for herbal products. Because of the exceptional growth in demand of herbal drugs, the required medicinal plants are randomly over exploited leading to scarcity or shortage of many valuable plant species. In India more than $90 \%$ plant species used by industry are collected from wild and over $60 \%$ of the collection involve destructive harvesting. According to an estimate over half a million tonnes of raw materials are indiscriminately collected from wild, mostly following destructive harvesting procedure and thus about 165,700 hectares forest being clear-felled each year. Hence alarming situations have resulted into short supply, high prices, forced import, or substitution and adulteration in crude drugs ${ }^{2}$ The reference of pratinidhi dravya is not seen in Brihatrayi except Vagbhata in Shodhanadi gana sangraha and other references are in Bhavaprakasha Nighantu, Yogaratnakara and Bhaishajyaratnavali but for scientists in contemporary times the substitution seems non-scientific and inappropriate, raising questions about validity of their use in treatment ${ }^{3}$. In ayurvedic 


\section{EPRA International Journal of Research and Development (IJRD)}

Volume: 6 | Issue: 10 | October 2021

- Peer Reviewed Journal

medicine Dasamula plants are top traded group and their annual demand is more than1000 Metric tons, Prsniparni moola is one among the Dasamula, Uraria picta is the original Prsniparni source while in trade or use Uraria lagopodioides (L.) DC, Desmodium gangeticum (L) DC, Pseudarthria viscida (L) Wight \& Arn are observed. Prsniparni is one of the most widely used herbs in Ayurvedic pharmaceutical industry where roots are mentioned in various formulations which results in destructive form of harvesting, so inorder to meet the high demand the drug is overexploited and also adultered and substituted with other drugs. Inorder to characterize and compare the raw drugs used as prsniparni in market, the candidate species traded under the name Prsniparni were subjected to macroscopic and organoleptic evaluation, microscopic study, Physical and phytochemical evaluation and High-performance thin layer chromatography (HPTLC)

\section{MATERIALS AND METHOD}

The present study was carried out under- the following headings:

- Collection of drugs

- Macroscopic study and organoleptic study

- Microscopic study (powder microscopy)

- Physico-chemical evaluation

- Phytochemical evaluation

Collection of drugs: Samples were collected from market and pharmacies of different parts of the country and were coded as:

Table: 1 Collection of Sample

\begin{tabular}{|c|c|}
\hline Sample & States \\
\hline 1. & Himachal Pradesh \\
\hline $\mathbf{2 .}$ & Maharashtra \\
\hline 3. & Karnataka \\
\hline 4. & Tamil Nadu \\
\hline 5. & Kerala \\
\hline
\end{tabular}

Place of study: Physico-Chemical and Phyto chemical were carried out in department of Dravyaguna, Sri Dharmasthala Manjunatheshwara College of Ayurveda and Hospital Hassan. Microscopy was carried out at Foundation for Revitalisation of Local health Traditions (FRLHT) Bangalore and High-performance thin layer chromatography (HPTLC) was done from Sri Dharmasthala Manjunatheshwara Research Centre for Ayurveda and Allied Science, Udupi.

Table: 2 Macroscopic study of Market Samples

\begin{tabular}{|c|c|c|c|c|c|}
\hline Features & Sample 1 & Sample 2 & Sample 3 & Sample 4 & Sample 5 \\
\hline Texture & Smooth & Smooth & Smooth & Smooth & Smooth \\
\hline Shape & Cylindrical & $\begin{array}{c}\text { Cylindrical and } \\
\text { twisted }\end{array}$ & $\begin{array}{c}\text { Cylindrical } \\
\text { and tortuous }\end{array}$ & $\begin{array}{c}\text { Cylindrical } \\
\text { and elongated }\end{array}$ & $\begin{array}{c}\text { Cylindrical } \\
\text { and elongated }\end{array}$ \\
\hline Shape & $\begin{array}{c}\text { Rough with } \\
\text { longitudinal } \\
\text { striations }\end{array}$ & Rough & Smooth & Rough & Rough \\
\hline Colour & Light yellow & Light brown & Dark brown & Dark brown, & Dark brown \\
\hline Odour & $\begin{array}{c}\text { Characteristic } \\
\text { herbaceous }\end{array}$ & Characteristic & $\begin{array}{c}\text { Saw dust } \\
\text { odour }\end{array}$ & Agreeable & Agreeable \\
\hline Fracture & Fibrous & Flexible & Brittle & Fibrous & Fibrous \\
\hline $\begin{array}{c}\text { Fracture } \\
\text { surface }\end{array}$ & Pale yellowish & Creamish & Creamish & Light brown & Light brown \\
\hline Taste & Slight acrid & Slight sweetness & Bitter & Slight sweet & Slight sweet \\
\hline
\end{tabular}

S 1-Himachal, S 2-Maharashtra, S 3-Karnataka, S 4-TamilNadu, S 5-Kerala 


\section{EPRA International Journal of Research and Development (IJRD)}

Table: 3 Microscopic findings of Market samples

\begin{tabular}{|c|c|c|c|c|c|}
\hline Features & Sample 1 & Sample 2 & Sample 3 & Sample 4 & Sample 5 \\
\hline Cork cells & Present & Present & Present & Present & Present \\
\hline Sclereids & Columnar & $\begin{array}{c}\text { Columnar } \\
\text { and rounded }\end{array}$ & $\begin{array}{c}\text { Columnar, small, } \\
\text { Thin walled }\end{array}$ & Thick walled & Thick walled \\
\hline Starch grains & Simple & $\begin{array}{c}\text { Simple } \\
\text { rounded }\end{array}$ & Simple rounded & Simple rounded & Simple rounded \\
\hline $\begin{array}{c}\text { Scalariform } \\
\text { vessels }\end{array}$ & $\begin{array}{c}\text { Long, broad } \\
\text { and pitted }\end{array}$ & Pitted and spiral & Spiral & Spiral \\
\hline
\end{tabular}

S 1-Himachal, S 2-Maharashtra, S 3-Karnataka, S 4-TamilNadu, S 5-Kerala

Table: 4 Powder Microscopy

\begin{tabular}{|c|c|c|c|c|c|}
\hline Features & Sample 1 & Sample 2 & Sample 3 & Sample 4 & Sample 5 \\
\hline Cork & Stratified & $\begin{array}{c}\text { Filled with } \\
\text { tannin } \\
\text { content }\end{array}$ & Thin walled \\
Stratified
\end{tabular}

S 1-Himachal, S 2-Maharashtra, S 3-Karnataka, S 4-TamilNadu, S 5-Kerala

Table: 5 Physicochemical evaluations

\begin{tabular}{|c|c|c|c|c|c|c|c|}
\hline SI NO & Physicochemical study & API & S 1 & S 2 & S 3 & S 4 & S 5 \\
\hline 1. & Foreign Matter & $\begin{array}{c}\text { Not more } \\
\text { than } 2 \%\end{array}$ & $1.5 \%$ & $1 \%$ & $1.8 \%$ & $2 \%$ & $2.5 \%$ \\
\hline 2. & Loss on drying & & $8.4 \%$ & $15 \%$ & $6.24 \%$ & $8.9 \%$ & $7.7 \%$ \\
\hline 3. & Total ash value & $\begin{array}{l}\text { Not more } \\
\text { than } 11 \% \\
\end{array}$ & $6 \%$ & $2.8 \%$ & $19.6 \%$ & $9 \%$ & $6.94 \%$ \\
\hline 4. & Acid insoluble ash & $\begin{array}{c}\text { Not more } \\
\text { than } 4 \%\end{array}$ & $2 \%$ & $.90 \%$ & $4.5 \%$ & $3.94 \%$ & $2.33 \%$ \\
\hline 5. & Water extractive value & $\begin{array}{l}\text { Not less } \\
\text { than } 8 \%\end{array}$ & $18.83 \%$ & $22.90 \%$ & $7.94 \%$ & $7.4 \%$ & $16.42 \%$ \\
\hline 6. & Alcohol extractive value & $\begin{array}{l}\text { Not less } \\
\text { than } 7 \%\end{array}$ & $7 \%$ & $4 \%$ & $3.89 \%$ & $3.37 \%$ & $2.93 \%$ \\
\hline
\end{tabular}

S 1- Himachal, S 2- Maharashtra, S 3- Karnataka, S 4- Tamil Nadu, S 5- Kerala 
EPRA International Journal of Research and Development (IJRD)

Volume: 6 | Issue: 10 | October 2021

- Peer Reviewed Journal

Table: 6 Phytochemical evaluation:

\begin{tabular}{|c|c|c|c|c|c|c|c|c|}
\hline SI NO & Test For & Test & Extract used & S 1 & S 2 & S 3 & S 4 & S 5 \\
\hline \multirow{4}{*}{1.} & \multirow{4}{*}{ Carbohydrates } & \multirow{2}{*}{ Fehling's test } & Water & + & + & + & + & + \\
\hline & & & Alcohol & + & + & - & + & - \\
\hline & & \multirow{2}{*}{ Benedict Test } & Water & + & + & - & + & + \\
\hline & & & Alcohol & + & + & - & - & - \\
\hline \multirow{6}{*}{2.} & \multirow{6}{*}{ Alkaloids } & \multirow{2}{*}{$\begin{array}{c}\text { Dragendroff's } \\
\text { Test }\end{array}$} & Water & - & - & - & - & - \\
\hline & & & Alcohol & + & - & - & - & + \\
\hline & & \multirow{2}{*}{ Wagner's Test } & Water & - & - & - & - & - \\
\hline & & & Alcohol & + & - & - & + & + \\
\hline & & \multirow{2}{*}{ Mayer's Test } & Water & - & - & - & - & - \\
\hline & & & Alcohol & + & - & - & + & + \\
\hline \multirow{4}{*}{3.} & \multirow{4}{*}{ Tannins } & Ferric chloride & Water & - & + & - & - & + \\
\hline & & Test & Alcohol & + & + & - & + & + \\
\hline & & Jead acetate & Water & - & - & - & - & + \\
\hline & & Lead acetate & Alcohol & + & - & - & + & + \\
\hline \multirow{2}{*}{4.} & \multirow{2}{*}{ Flavonoid } & \multirow{2}{*}{ Lead acetate } & Water & + & + & - & + & + \\
\hline & & & Alcohol & + & + & + & + & + \\
\hline \multirow{2}{*}{5.} & \multirow{2}{*}{ Glycosides } & \multirow{2}{*}{ Cardiac glycosides } & Water & + & + & - & + & + \\
\hline & & & Alcohol & + & - & + & + & + \\
\hline \multirow{2}{*}{6.} & \multirow{2}{*}{ Steroids } & \multirow{2}{*}{$\begin{array}{c}\text { Salkawski } \\
\text { reaction }\end{array}$} & Water & + & + & - & + & + \\
\hline & & & Alcohol & + & + & - & + & + \\
\hline \multirow{2}{*}{7.} & \multirow{2}{*}{ Phenols } & & Water & + & - & - & - & - \\
\hline & & & Alcohol & + & - & - & - & - \\
\hline \multirow{2}{*}{8.} & \multirow{2}{*}{ Coumarins } & & Water & - & - & - & - & - \\
\hline & & & Alcohol & - & - & - & - & - \\
\hline \multirow{2}{*}{9.} & \multirow{2}{*}{ Triterpenoids } & & Water & + & + & - & - & - \\
\hline & & & Alcohol & + & + & - & - & - \\
\hline \multirow{2}{*}{10.} & \multirow{2}{*}{ Carboxylic acid } & & Water & - & - & - & - & - \\
\hline & & & Alcohol & - & - & - & - & - \\
\hline & & & Water & + & + & - & + & + \\
\hline 11. & Saponins & & Alcohol & + & + & + & + & + \\
\hline 12 & Ouingnes & & Water & - & + & - & - & - \\
\hline 12. & Qumones & & Alcohol & - & + & - & - & - \\
\hline
\end{tabular}

S 1-Himachal, S 2-Maharashtra, S 3-Karnataka, S 4-TamilNadu, S 5-Kerala

\section{OBSERVATION}

As per section 9 A of the drug and cosmetic act, 1940 defines an adultered drug as the one containing any harmful or toxic substance which may be injurious to health; or if any substance has been mixed with it so as to reduce its quality or strength ${ }^{4}$. The results of organoleptic evaluation, Microscopic evaluation ,powder microscopy, Physico-phytochemical evaluation of the market samples to that of genuine sample and also the standards mentioned in API evidenced that the majority of market samples were unintentionally adultered and also some samples were substitutes as per database. As per the microscopic evaluation; sample 3 which was Pseudarthria viscida and also sample 4 and 5 was identified as Uraria lagopodiodes which was mentioned by database as substitutes, but sample 2 was entirely a different drug which was not a substitute but can be taken as adulterant and was identified as Vigna mungo. All the market sample were showing almost similar properties in Physico-chemical evaluation .

\section{DISCUSSION}

Through the present study its evident that what is received from the market by the name of Prsniparni are not the original one its either substitute or adulterant. Almost all the substitute is dissimilar taxonomically as they belong to different families or species, but majority of the species which was received for the study was substitute which was a better option than adulterants. By using substitutes the pressure over a single drug can be reduced. The most important criteria for the selection of pratinidhi seem to be similarity in indications \& 


\section{EPRA International Journal of Research and Development (IJRD)}

Volume: 6 | Issue: 10 | October 2021

- Peer Reviewed Journal

Pharmaco-therapeutic uses. Finally, the study reveals that the status of the commercially available crude drug which was received as prsniparni was having less quality and purity and the materials received from markets were either substitute or adulterant, also care should be taken during the time of harvest

Being an unavoidable drug among Dasamoola with wide spectrum of uses, the substitution of Prsniparni should be judicial and authorised after conducting detailed scientific evaluation studies. Substitution with other useful plant or part is one of the early signs of upcoming extinction. Therefore, judicious usage and cultivation should be initiated for common medicinal species having huge consumption

\section{CONCLUSION}

The present study was Comparative Pharmacognostic evaluation of market samples of Prsniparni with genuine source of Uraria picta (jacq.) Desv. ex DC

- The objective of the study was pharmacognostic evaluation of market samples of Prsniparni and compare with natural habitat sample of Uraria picta

- Since the genuine root samples were not available, the Pharmacies were overcoming the situation mainly by substituting the drug with available substitutes. ${ }^{5}$

- Four samples were collected from different market across the country and compared with the sample collected from natural habitat, Sirmour district of Himachal Pradesh.

- The present study showed that the four market samples are not the original source drug they are either substituted or adulterated and possess less quality and purity.

As per the study conducted the samples received to be concluded as

Table: 7 Identification of Market samples

\begin{tabular}{|c|c|c|}
\hline Sample No & Place of collection & Source Plant \\
\hline 1. & Himachal Pradesh & Uraria picta (jacq.) Desv. ex DC \\
\hline 2. & Pune & Vigna mungo (L.) Hepper \\
\hline 3. & Karnataka & Pseudarthria viscida (L.) Wight \& Arn. \\
\hline 4. & Tamilnadu & Uraria lagopodioides (L.) DC \\
\hline 5. & Kerala & Uraria lagopodioides (L.) DC \\
\hline
\end{tabular}

\section{LIMITATIONS}

The study was carried out in finest possible manner by managing the time. But still there were limitations.

The limitation faced during the study was:

- $\quad$ HPTLC should have been done by comparing with marker compound.

\section{REFERENCES}

1. S. Lalitha, Pisharath Madhavan Deepthi, Sebastian John Adams, Kulithalai V Krishnamurthy: Comparative Pharmacognosy of Medicinal Plant species used as Prsniparni. International Journal of Green Pharmacy. Int J Green Pharm, 2012; Vol 6 (4):303-309

2. Raghavendra Naik, Shubhashree M.N: An insight to the current Substitutes and Adulterants, Souveneir of Dravyaguna Prabhodini, Feb 2019, Government Ayurveda Medical College, Bangalore, p.49.

3. Raghavendra Naik, Shubhashree M.N: An insight to the current Substitutes and Adulterants, Souveneir of Dravyaguna Prabhodini, Feb 2019, Government Ayurveda Medical College, Bangalore, p.50.

4. Arnab Battacharya, Animesh K.Datta:Uraria picta An overview of Medicinal And Aromatic Plant Science and Biotechnology,2010.

5. Depletion of genuine raw drugs: A rising threat in Ayurvedic drug Manufacturing Industry 


\section{ANNEXURES}

Figure: 1 Macroscopy of market samples

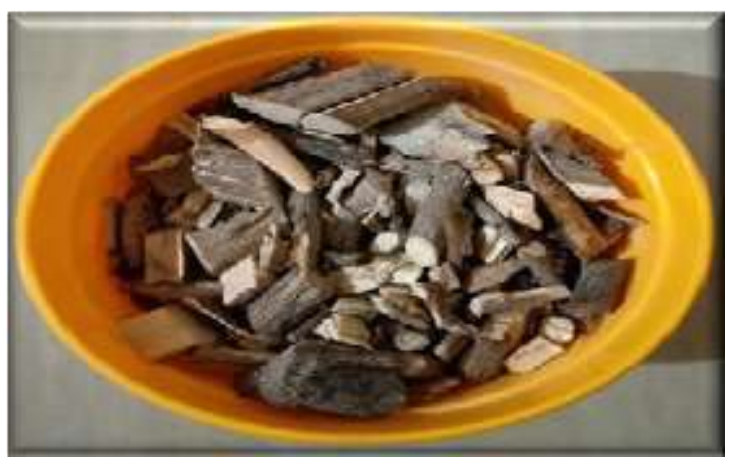

Sample 1

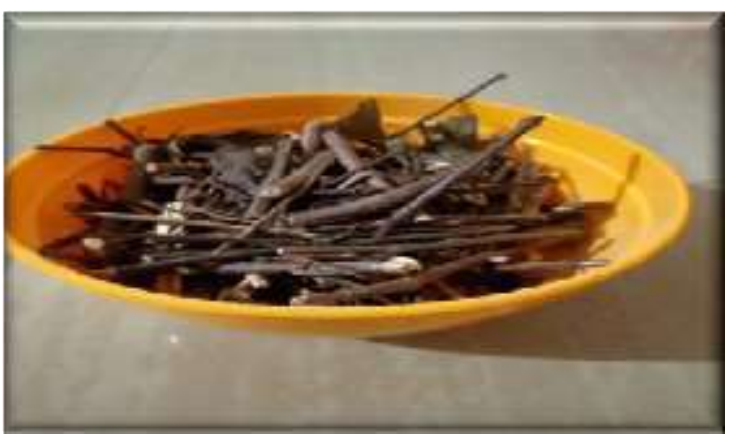

Sample 3

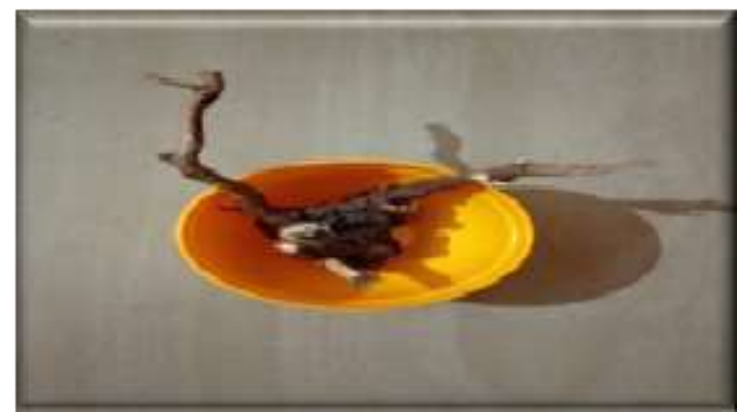

Sample 5

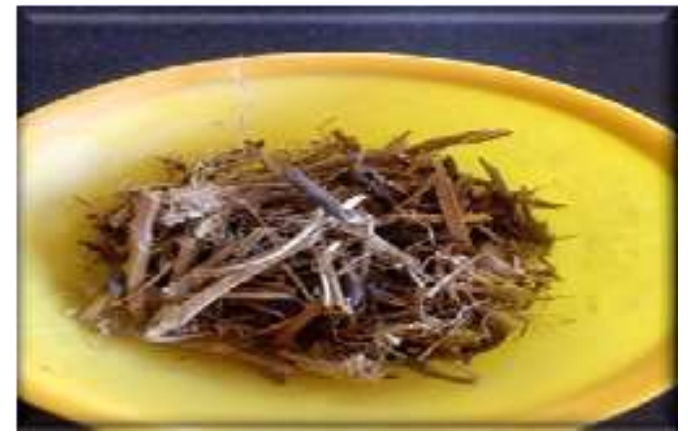

Sample 2

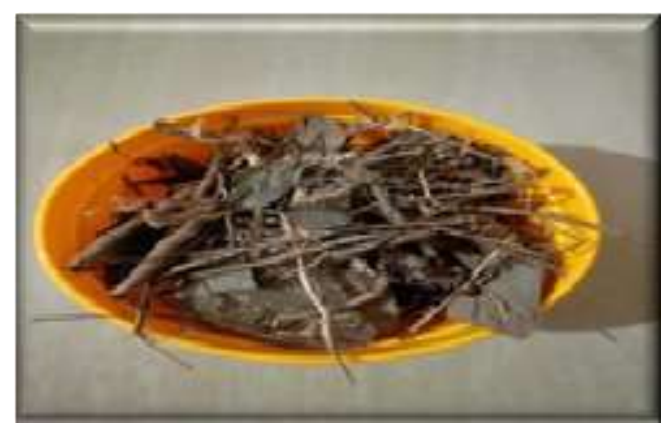

Sample 4 
Figure: 2 Powder microscopy

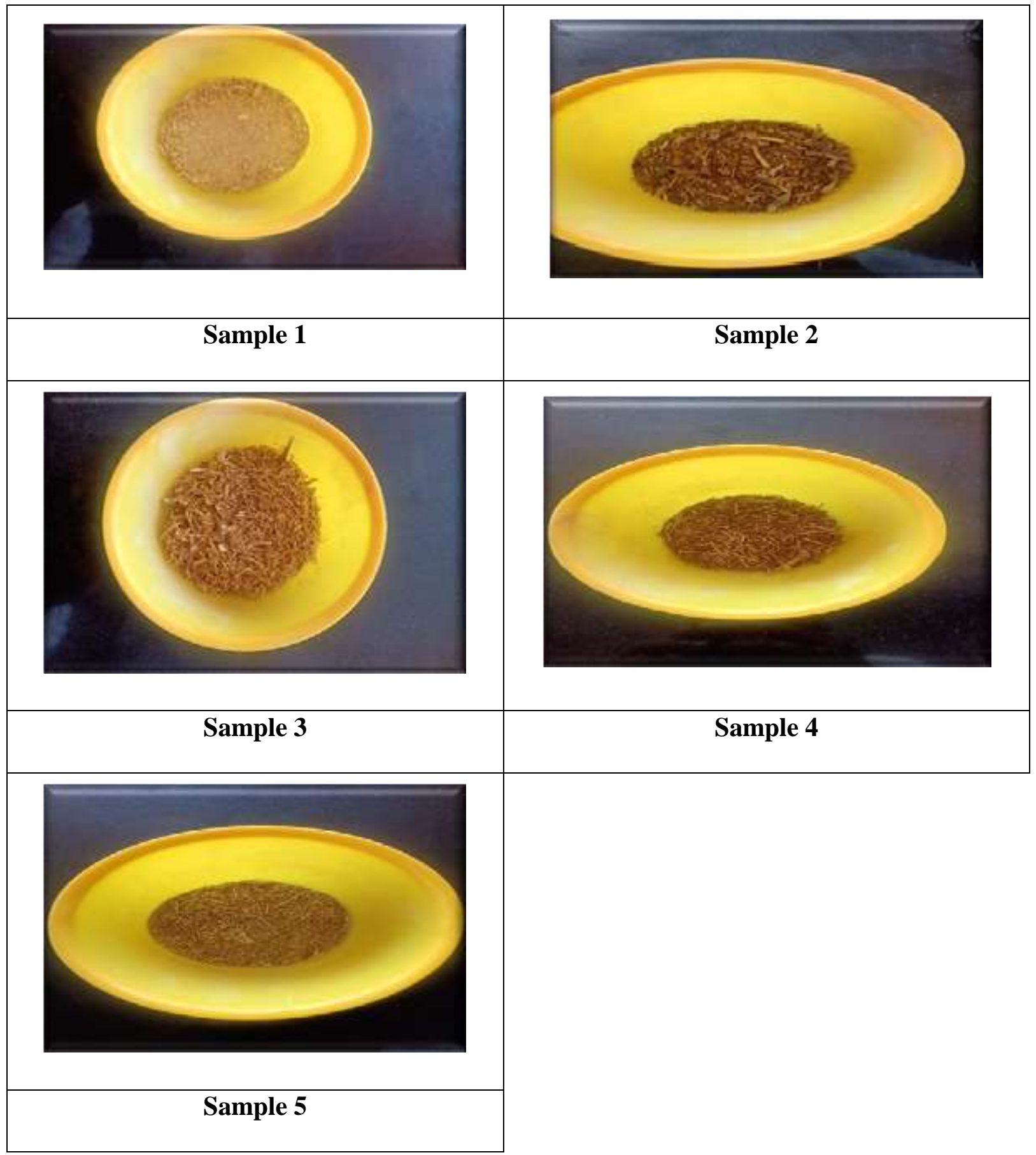


Figure: 3 Microscopy Sample 1
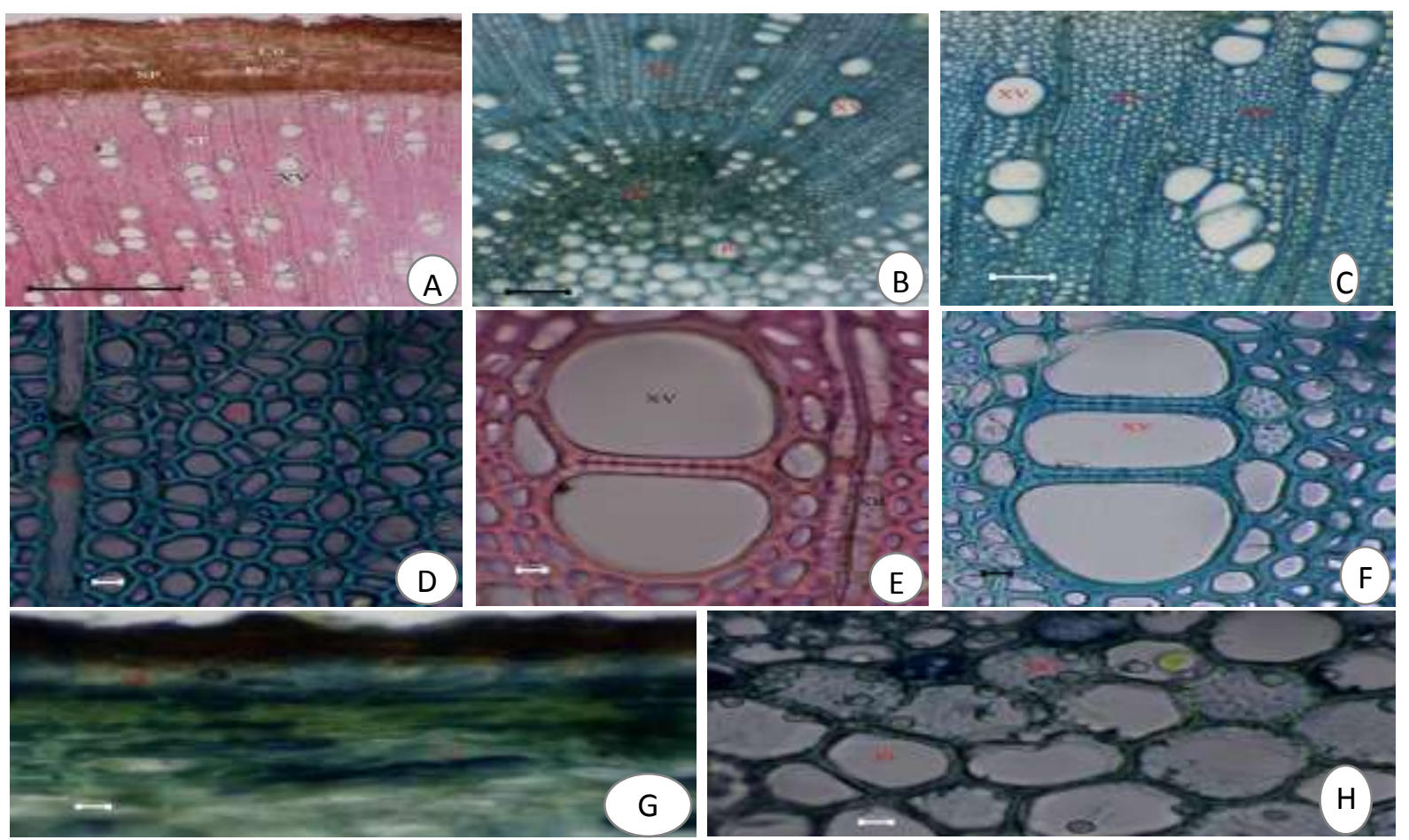

Co - Cortex; Ck - Cork; Fi -Fibres; Pi - Pith; SG - Starch Grains; SP - Secondary Phloem; SXV - Scalariform Xylem vessels; Ta - Tannin; XF - Xylem Fibre; XR Xylem Rays; XV - Xylem Vessels 


\section{EPRA International Journal of Research and Development (IJRD)}

Figure:4 Powder Microscopy Sample 1
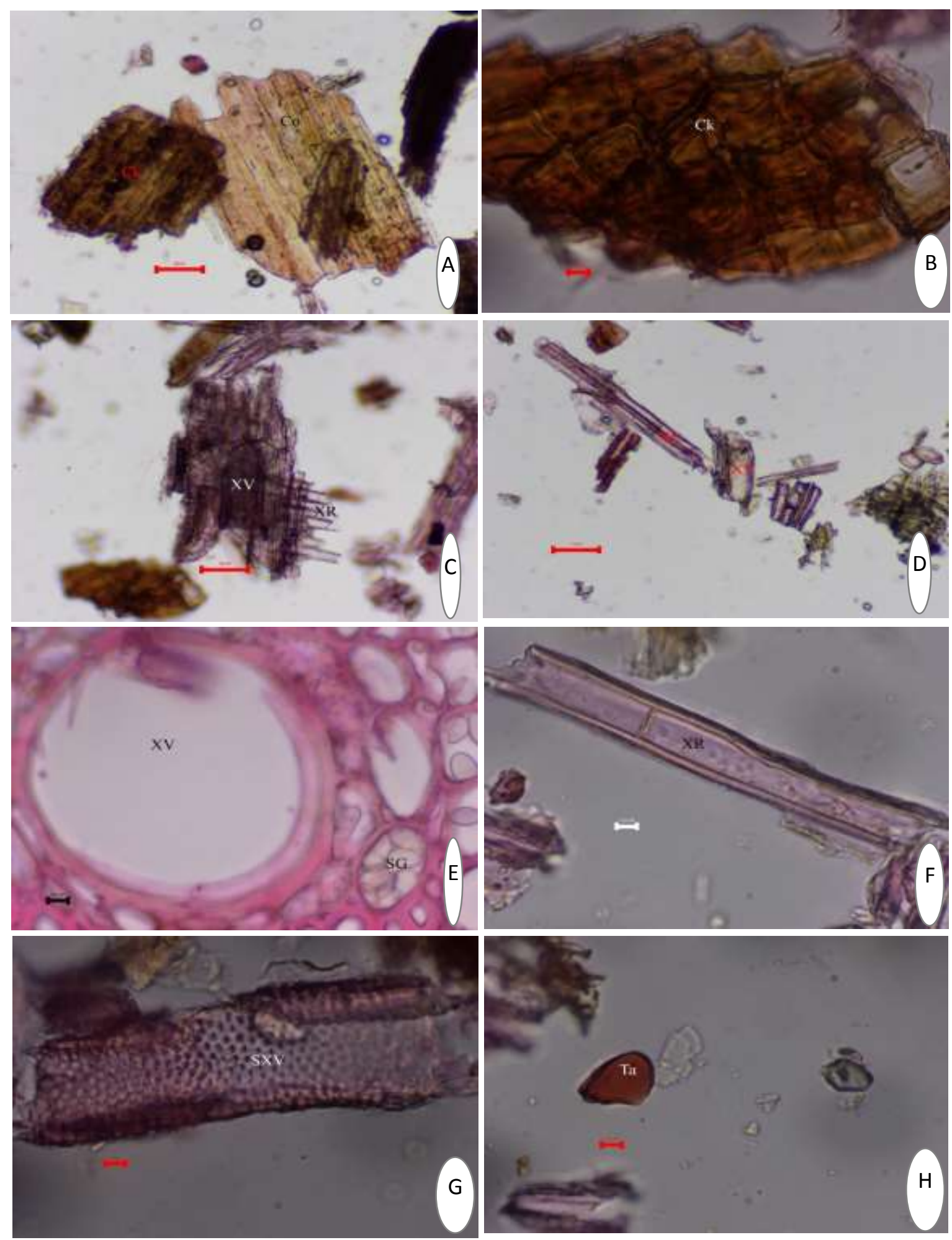
EPRA International Journal of Research and Development (IJRD)

Figure: 5 Microscopy Sample 2
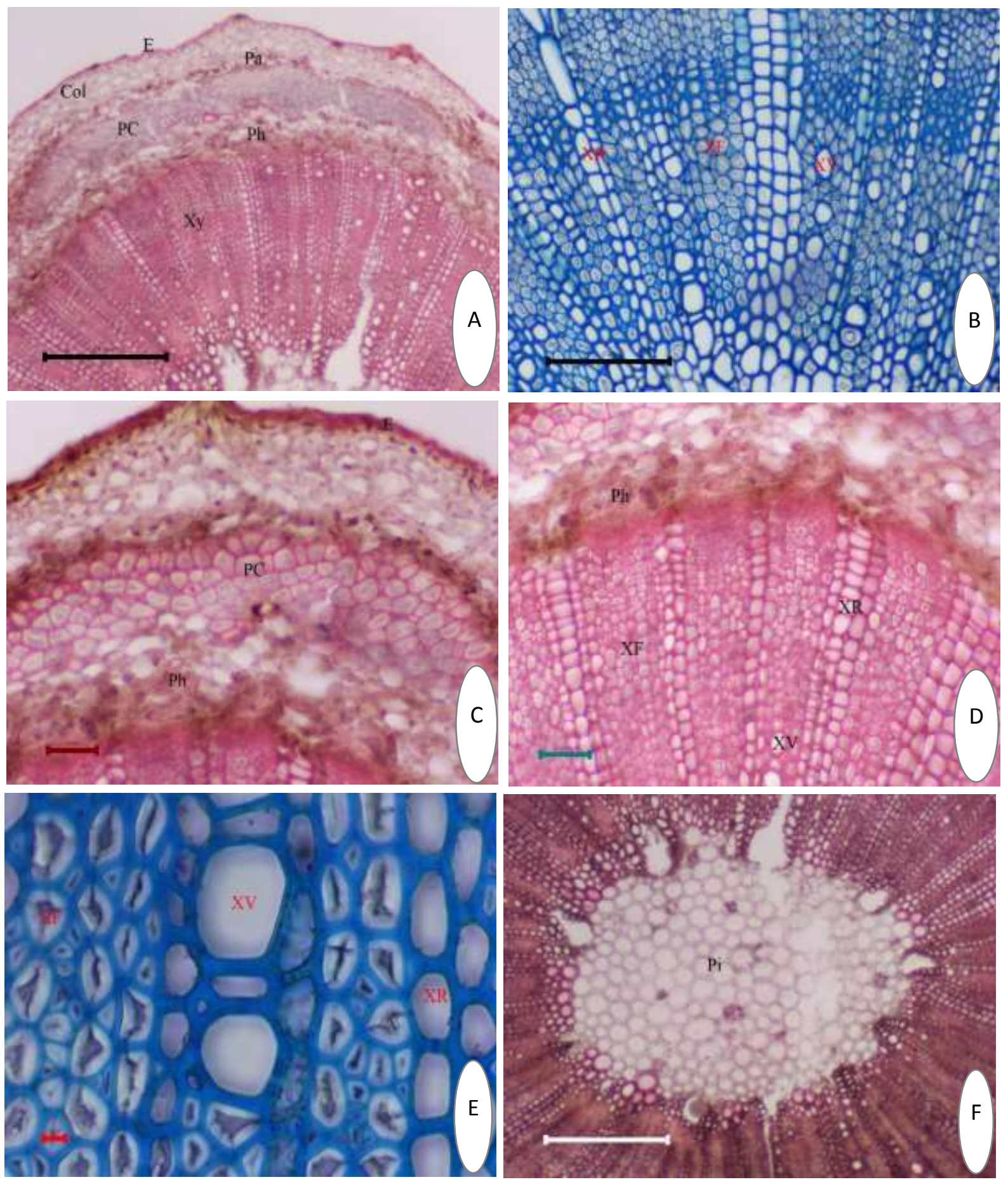

Co - Cortex; Col - Collenchyma; E - Epidermis; Pa - Parenchyma; PC - Pericycle; Ph - Phloem; Pi - Pith; SG - Starch Grains; ST - Stone cells; SXV - Scalariform Xylem vessels; Ta - Tannin; XF - Xylem Fibre; XR - Xylem Rays; XV - Xylem Vessels; Xy Xylem 


\section{(e)}

SJIF Impact Factor 2021: 8.013| ISI I.F.Value:1.241| Journal DOI: 10.36713/epra2016 ISSN: 2455-7838(Online)

EPRA International Journal of Research and Development (IJRD)

Volume: 6 | Issue: 10 | October 2021

- Peer Reviewed Journal

Figure: 6 Powder microscopy Sample 2
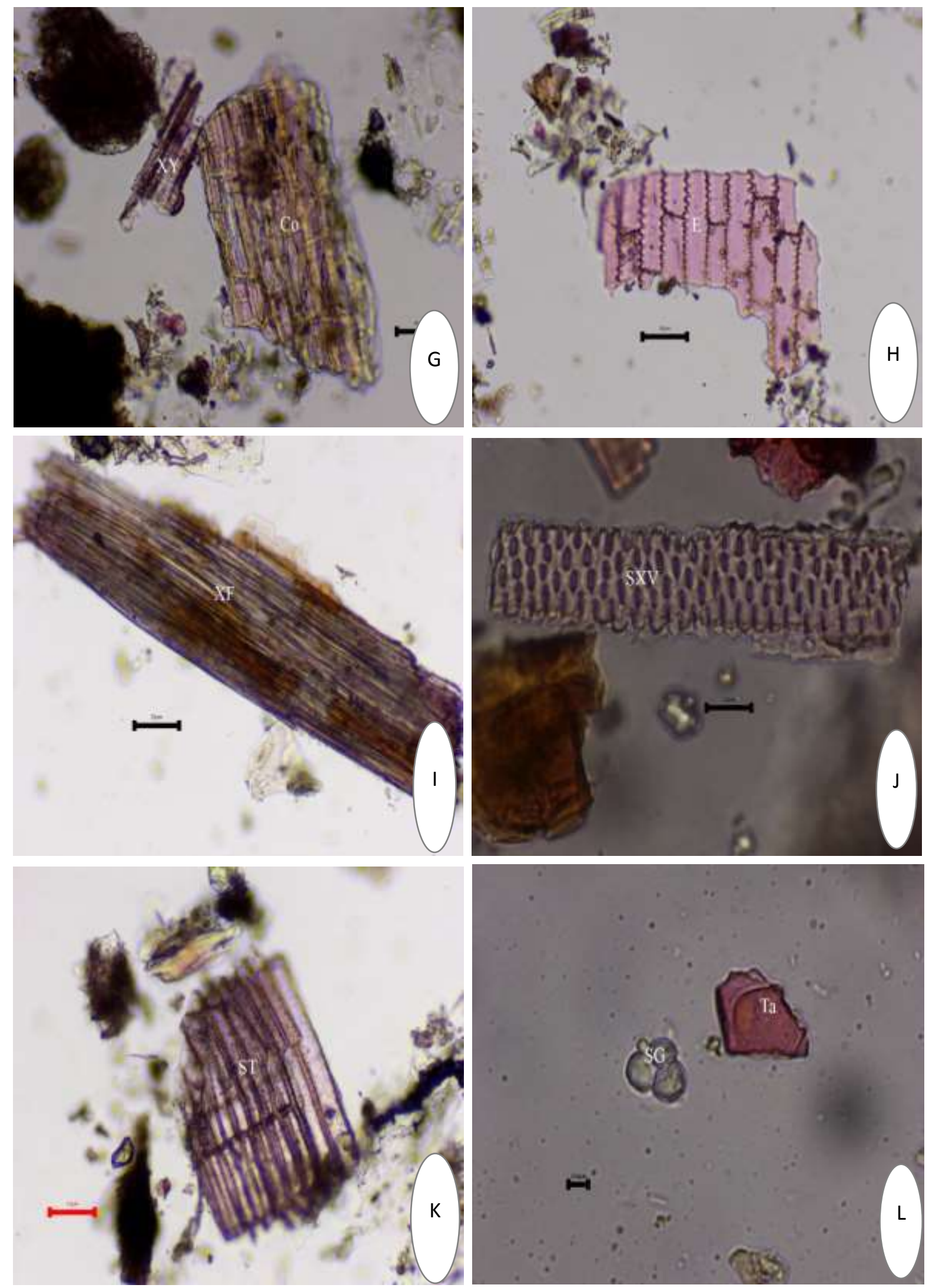

(c) 2021 EPRA IJRD 
EPRA International Journal of Research and Development (IJRD)

Figure:7 Microscopy Sample 3
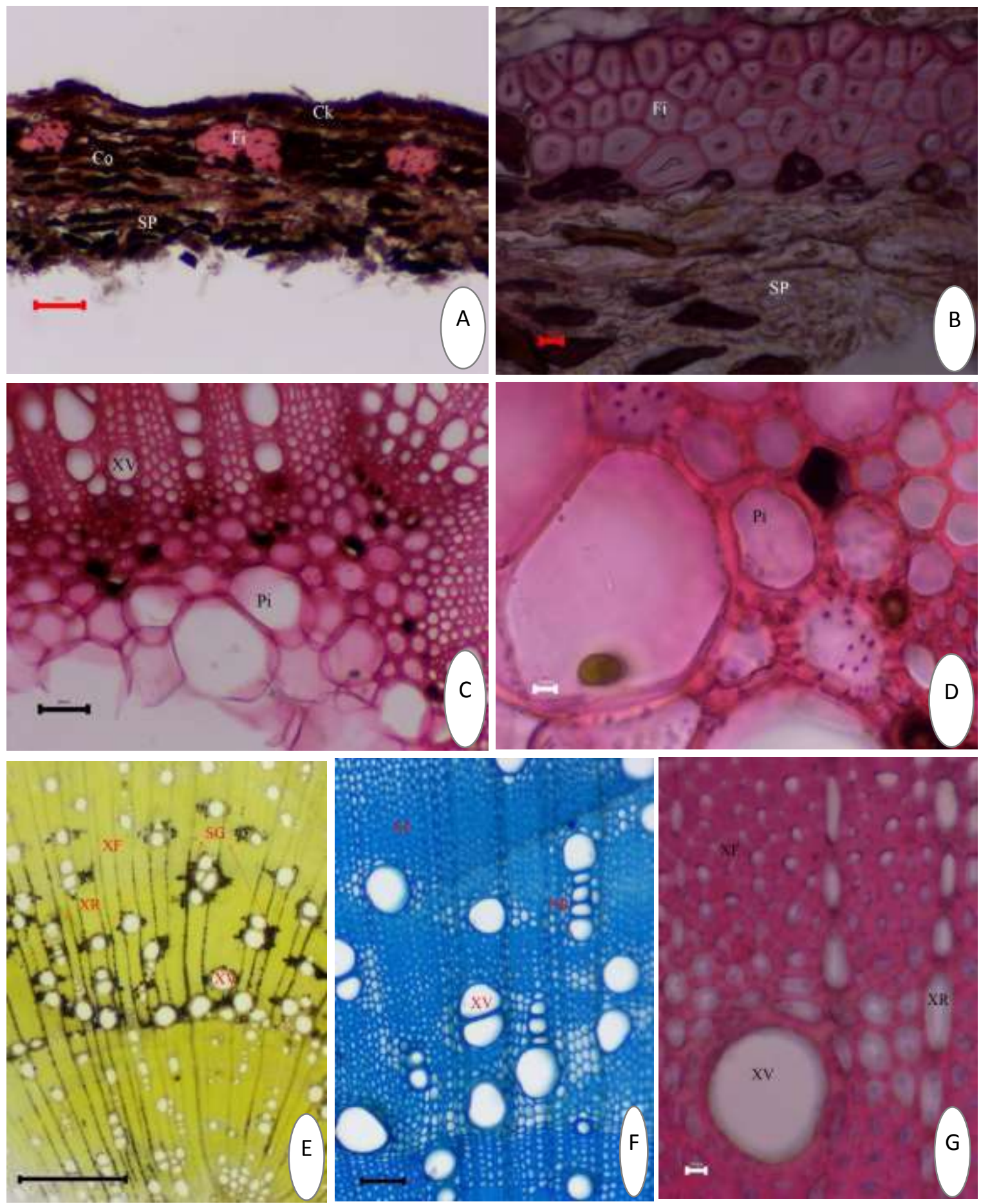

Co - Cortex; Ck - Cork; Fi - Fibres; Ph C - Phenolic Compound; Pi - Pith; SG Starch Grains; SP - Secondary Phloem; SXV - Spiral Xylem Vessels; SXV Scalariform Xylem vessels; Ta - Tannin; XF - Xylem Fibre; XR - Xylem Rays; XV Xylem Vessels 
Figure: 8 Powder microscopy Sample 3
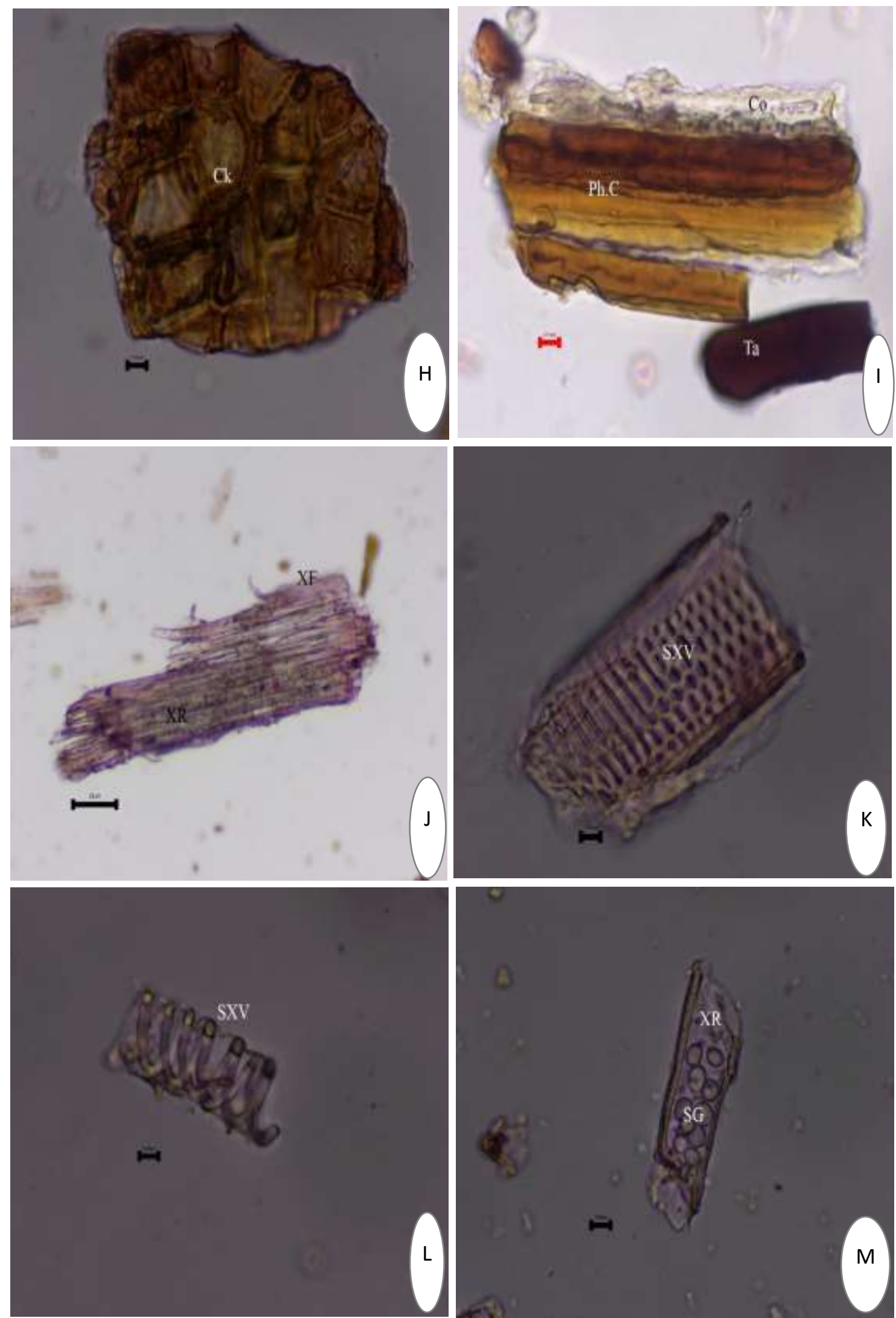
Figure: 9Microscopy Sample 4
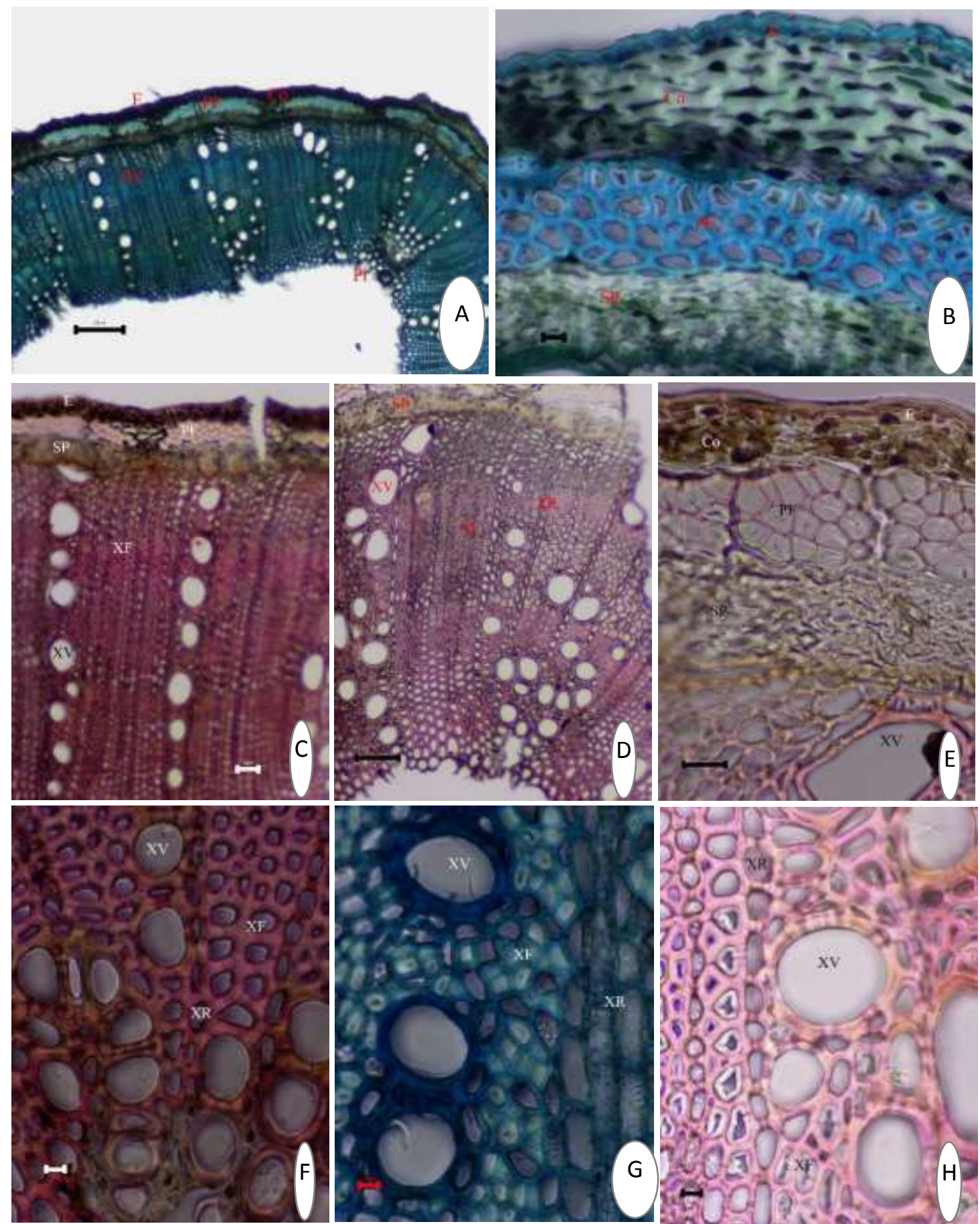

Co - Cortex; Ck - Cork; Fi - Fibres; SX - Secondary xylem; SP - Secondary Phloem; XF

- Xylem Fibre; XR - Xylem Rays; XV - Xylem Vessels; XCF - Xylem crystal fibres 
EPRA International Journal of Research and Development (IJRD)

Figure: 10 Powder microscopy Sample 4

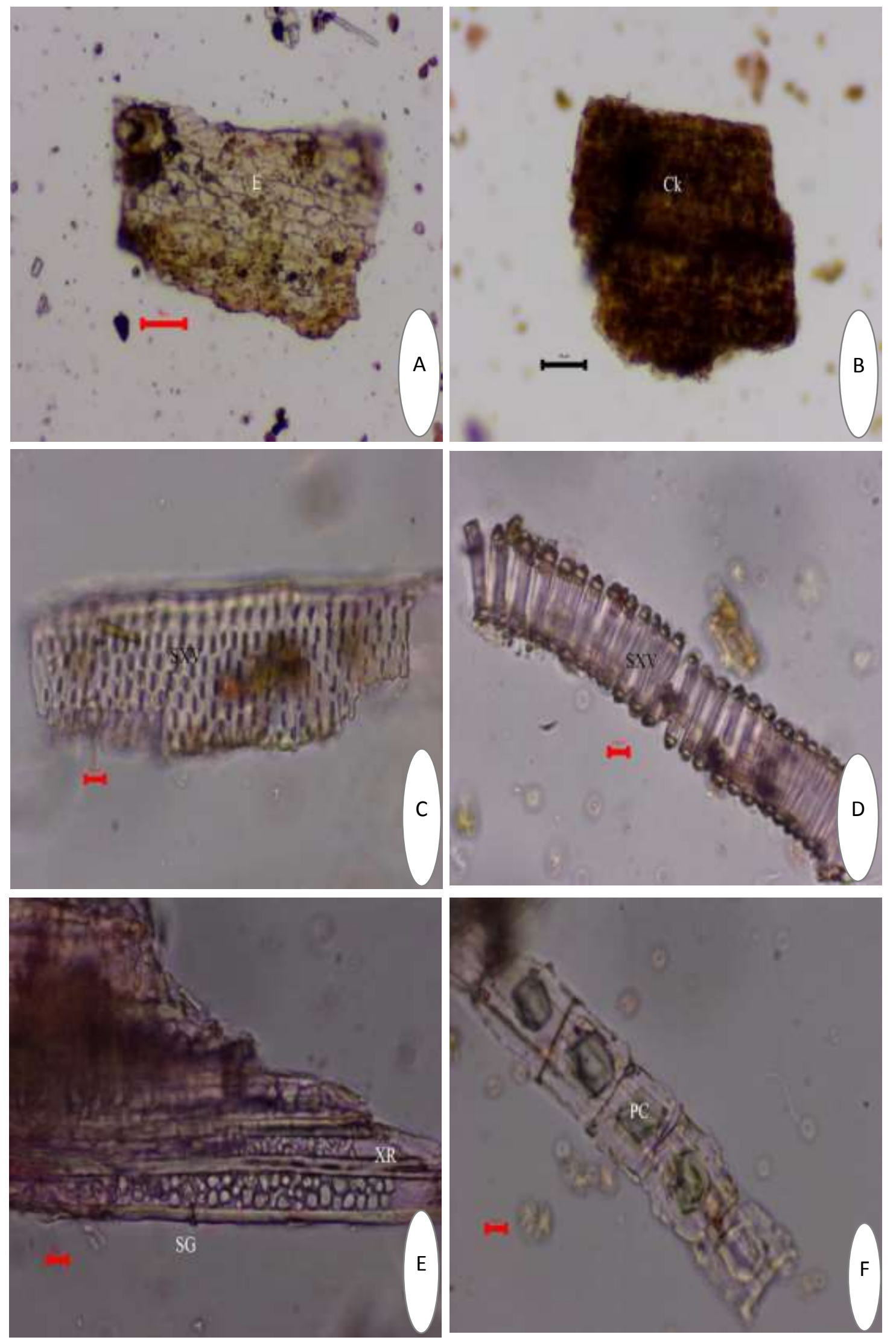

(c) 2021 EPRA IJRD | Journal DOI: https://doi.org/10.36713/epra2016 | www.eprajournals.com |299 | 


\section{(s)}

SJIF Impact Factor 2021: 8.013| ISI I.F.Value:1.241| Journal DOI: 10.36713/epra2016 ISSN: 2455-7838(Online)

EPRA International Journal of Research and Development (IJRD) Volume: 6 | Issue: 10 | October 2021

- Peer Reviewed Journal

Figure: 11 Microscopy Sample 5
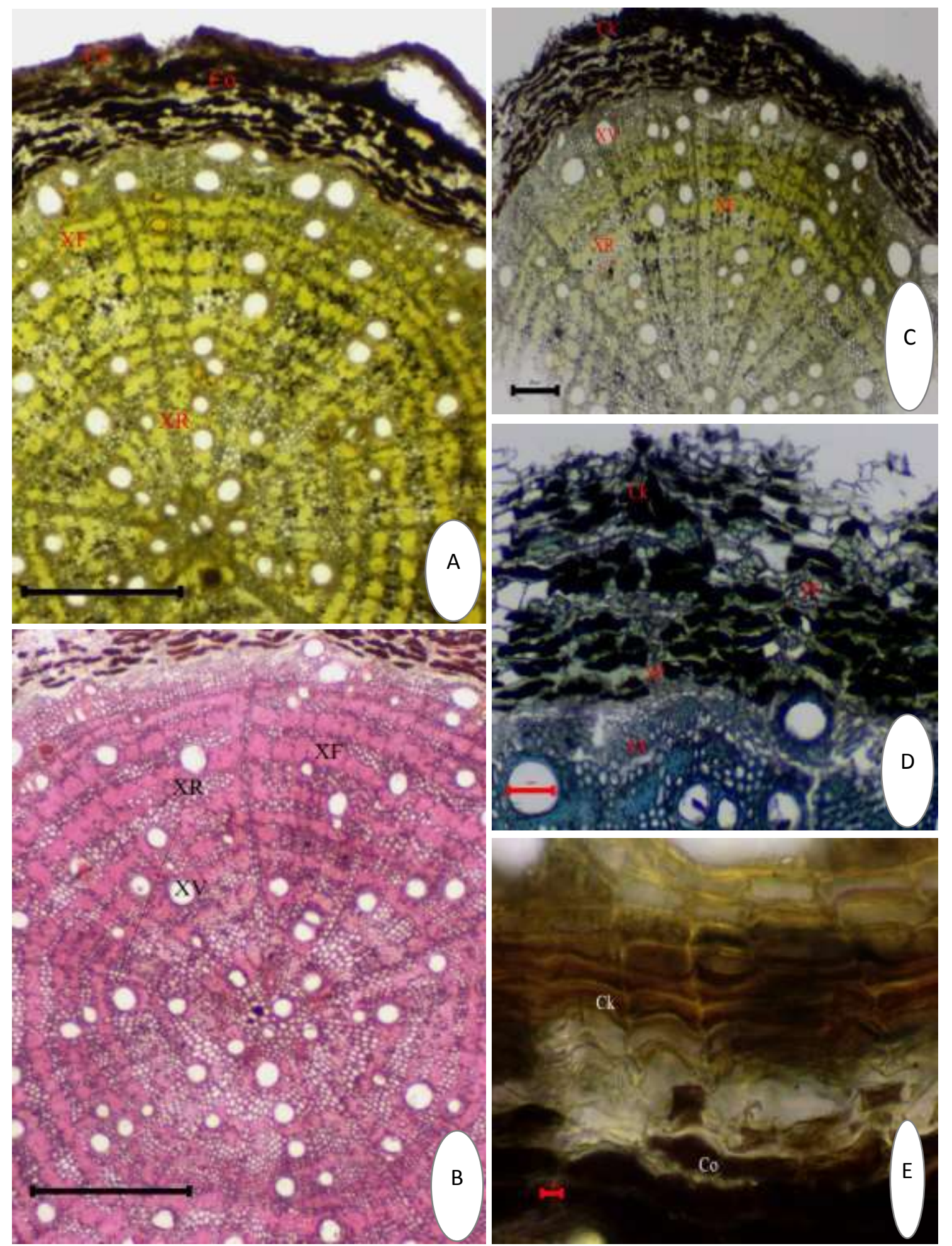

BC - Brown content; Ck - Cork; Co - Cortex; PC - Prismatic crystals; Ph C - Phenolic content; SG - Starch Grains; SXV - Scalariform Xylem vessels; XR - Xylem Rays 
EPRA International Journal of Research and Development (IJRD) Volume: 6 | Issue: 10 | October 2021

- Peer Reviewed Journal

\section{Figure:12 Powder microscopy Sample 5}

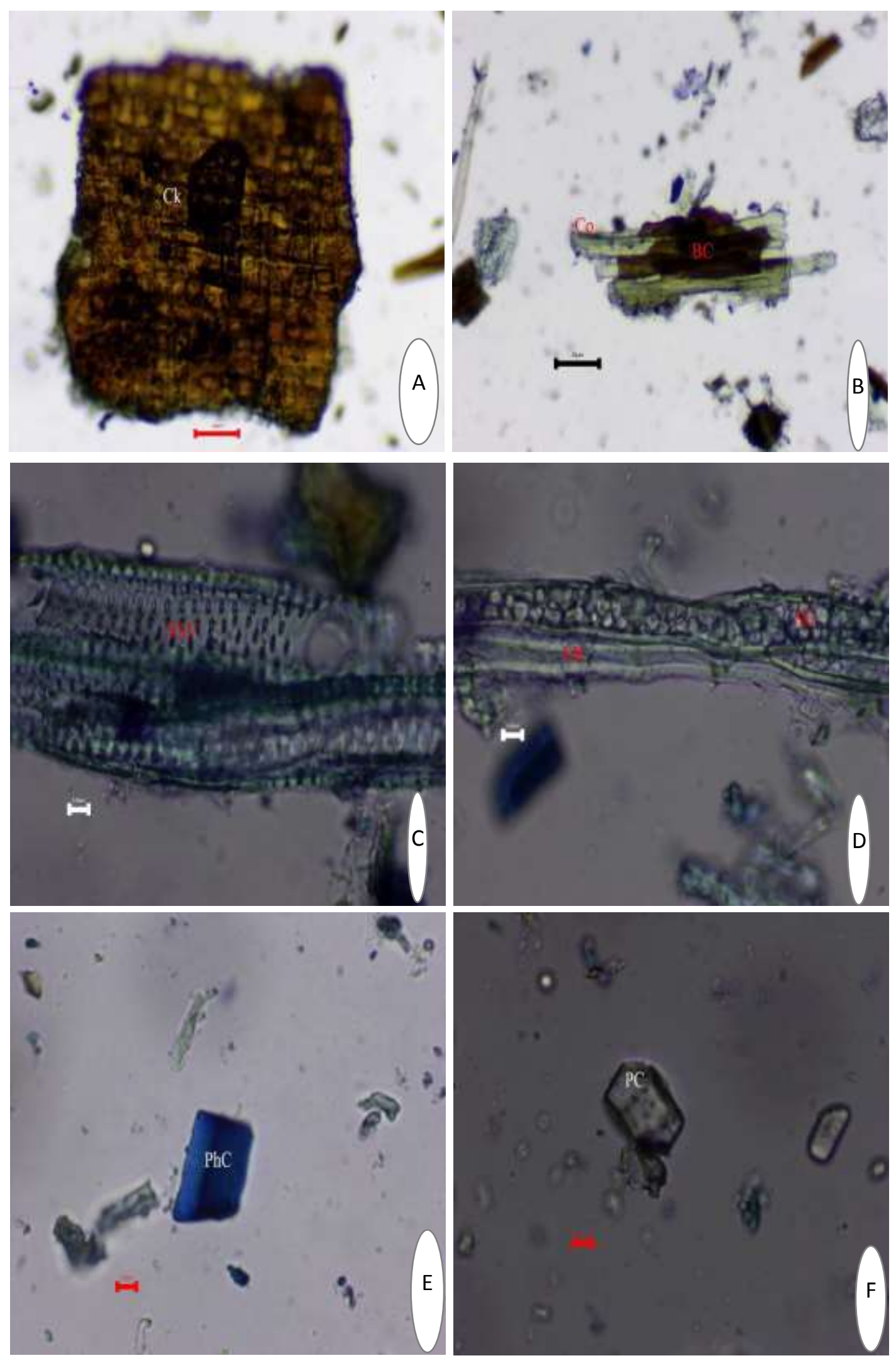

(c) 2021 EPRA IJRD | Journal DOI: https://doi.org/10.36713/epra2016 | www.eprajournals.com |301 | 